(LNSC), and overnight dexamethasone suppression test (DST). ${ }^{2}$ We sought to evaluate the validity of anecdotal reports that morning or random serum cortisol, which lacks sensitivity and specificity and is not recommended for this purpose, is commonly used to test for hypercortisolism in primary care. We analysed test selection preferences for hypercortisolism in patients with adrenal incidentalomas or suspected secondary hypertension in primary care, using data from our communitybased laboratory. We extracted requests for any of the aforementioned cortisol tests with metanephrines or aldosterone and renin within one month of each other, over a 12-month period. We then reviewed request forms to select only those explicitly testing for secondary hypertension or functionality of an adrenal incidentaloma, yielding 175 cases. Serum cortisol was the most common test ( $81 \%$ of cases; $95 \%$ CI $74.9-100 \%)$. There is a significant opportunity for pathologists to provide education to general practitioners (GP) regarding initial testing for hypercortisolism. We intend repeating this analysis after a focused education campaign incorporating GP detailing, printed and electronic materials, and enhanced interpretative commenting.

References

1. Lacroix A, Feelders RA, Stratakis CA, et al. Cushing's syndrome. Lancet 2015; 386: 913-27.

2. Nieman LK, Biller BM, Findling JW, et al. The diagnosis of Cushing's syndrome: an Endocrine Society Clinical Practice Guideline. J Clin Endocrinol Metab 2008; 93: 1526-40.

\section{PSEUDOHYPOALDOSTERONISM TYPE 2 WITH CUL3 MUTATION CONFIRMED FOLLOWING RETESTING 15 YEARS LATER}

${\underline{B o b b y ~} \mathrm{Li}^{1,2}}$, Steven Soule ${ }^{3}$, Chris Florkowski ${ }^{1,2}$, Thomas Wilkinson ${ }^{3}$

${ }^{1}$ Canterbury Health Laboratories, Christchurch, New Zealand; ${ }^{2}$ Department of Pathology, University of Otago, Christchurch, New Zealand; and ${ }^{3}$ Department of Endocrinology, Canterbury District Health Board, Christchurch, New Zealand

This work was done at Canterbury Health Laboratories, Christchurch, New Zealand.

A 22-year-old Caucasian female presented to endocrinology clinic in late 2004 for investigation of early-onset hypertension, with systolic blood pressure $180-200 \mathrm{mmHg}$ and diastolic blood pressure of $110-120 \mathrm{mmHg}$. She was hyperkalaemic $(5.4 \mathrm{mmol} /$ L) with an elevated aldosterone:renin ratio $[1663 \mathrm{pmol} / \mathrm{L} /(\mathrm{nmol} /$ L/h); URL 750] and metabolic acidosis with $\mathrm{pH} 7.26$ and bicarbonate $20.6 \mathrm{mmol} / \mathrm{L}$. Pseudohypoaldosteronism Type 2 (PHA2) or Gordon's Syndrome was diagnosed. Mutation testing for $W N K 1$ and $W N K 4$ was negative. She had variable adherence to bendrofluazide and salt restriction and was lost to follow up before re-presentation with congestive heart failure in 2020 . Massive parallel sequencing with a pseudohypoaldosteronism panel identified heterozygosity of the CUL3 gene for c.1207$12 \mathrm{~T}>\mathrm{A}$, an intra-intronic variant predicted to disrupt splicing, deemed likely pathogenic, seen previously in an Australian kindred with PHA2. Although our patient's initial genetic testing was negative in 2005, advances in knowledge of PHA2 led to a satisfactory genetic diagnosis 15 years later. This demonstrates that when an inherited condition is suspected, even when previous genetic testing is negative, there may be utility in revisiting genetic testing if knowledge of the condition has improved and subsequent testing can evaluate more genes or variants than the initial genetic test.

\section{HB WESTPORT [HBB: C.366A>C (P.GLU122ASP)], A NOVEL $\beta$-GLOBIN VARIANT INTERFERING WITH HBA1C}

Jordyn Moore ${ }^{1}$, Bobby $\mathrm{Li}^{1,2}$, Darrell Wang ${ }^{3}$, Becca Chan ${ }^{1}$, Richard King ${ }^{1,2,3}$, Chris Florkowski ${ }^{1,2}$

${ }^{1}$ Specialist Biochemistry, Canterbury Health Laboratories, Christchurch, New Zealand; ${ }^{2}$ Department of Pathology, University of Otago, Christchurch, New Zealand; and ${ }^{3}$ Molecular Pathology, Canterbury Health Laboratories, Christchurch, New Zealand

This work was done at Canterbury Health Laboratories, Christchurch, New Zealand.

Due to increasing measurement of $\mathrm{HbAlc}$ for diagnosis and management of diabetes, haemoglobin $(\mathrm{Hb})$ variants are routinely detected during $\mathrm{HbA} 1 \mathrm{c}$ measurement by high performance liquid chromatography (HPLC). A 45-year-old Caucasian female was referred for blood testing to investigate fatigue, with $\mathrm{HbA} 1 \mathrm{c}$ included to screen for diabetes. Abnormal HbA1c peak tailing on Bio-Rad D-100 cation exchange HPLC was observed, with $\mathrm{HbA} 1 \mathrm{c}$ of $52 \mathrm{mmol} / \mathrm{mol}(6.9 \%)$ calculated. However, HbA1c by DCA Vantage Point-of-Care Analyzer immunoassay was $34 \mathrm{mmol} / \mathrm{mol}$ (5.3\%). Electrospray ionisation (ESI) time-of-flight (TOF) mass spectrometry demonstrated both normal $(15,867 \mathrm{Da})$ and variant $\beta$-globin $(15,853 \mathrm{Da})$ masses, with the variant chain $48 \%$ of total $\beta$-globin. DNA sequencing confirmed the patient was heterozygous for $[H B B$ : c.366A $>$ C (p.Glu122Asp)], which has not previously been described. Consistent with reports for other variants at the same amino acid position, our patient had a clinically normal phenotype, with unremarkable full blood examination during pregnancy. Glucose based criteria was recommended for any future diabetes screening due to interference from the novel variant, which causes overestimation of $\mathrm{HbA} 1 \mathrm{c}$ by routine HPLC methods.

\section{THE AGE OF IRON: AN ASSESSMENT OF AGE- RELATED DISTRIBUTION IN PAEDIATRIC IRON STUDIES}

Marcela P. Nascimento ${ }^{1}$, Louise Prentice ${ }^{2,3,4}$,

Michael B. Theophilos ${ }^{1}$, Catherine Lynch ${ }^{1}$, Daniella Angeleski ${ }^{1}$, Ken A. Sikaris

${ }^{1}$ Melbourne Pathology, Melbourne, Vic, Australia; ${ }^{2}$ Hobart Pathology, Hobart, Tas, Australia; ${ }^{3}$ Royal Hobart Hospital, Hobart, Tas, Australia; and ${ }^{4}$ University of Tasmania, Hobart, Tas, Australia

Introduction: Clinical decision limits and reference limits for ferritin and transferrin saturation (TSAT) are used for the investigation of iron deficiency (ID) and iron overload (IO). Our aim is to assess the distribution of ferritin, transferrin and TSAT in paediatric populations from two private laboratory services. Methods: 315,149 iron studies results for patients under 18 years collected between 2010 and 2020 were extracted from our database. All tests were measured using the Roche Cobas 8000 immunoturbidimetric method. Results were excluded if the 